\title{
ECHOCARDIOGRAPHIC AND CARDIAC SINGLE PHOTON EMISSION COMPUTED TOMOGRAPHY PREDICTORS OF LEFT VENTRICLE REVERSE REMODELING AFTER SURGICAL REVASCULARIZATION IN PATIENTS WITH ISCHEMIC CARDIOMYOPATHY AND LEFT VENTRICLE SYSTOLIC DYSFUNCTION
}

\author{
Martin Hutyra $^{\mathrm{a} *}$, Tomas Skala ${ }^{\mathrm{a}}$, Milan Kaminek ${ }^{\mathrm{b}}$, Petr Nemec ${ }^{\mathrm{c}}$ \\ ${ }^{a}$ 1. Department of Internal Medicine Teaching Hospital Olomouc, Czech Republic \\ ${ }^{b}$ Department of Nuclear Medicine, Teaching Hospital Olomouc \\ c Department of Cardiosurgery, Teaching Hospital Olomouc \\ e-mail:martinhutyra@seznam.cz
}

Received: March 15, 2008; Accepted: May 3, 2008

Key words: Heart failure/Left ventricle systolic dysfunction/Ischemic cardiomyopathy/Reverse remodeling prediction/ Echocardiography/Cardiac gated SPECT

Background: The extent of scar or viable hypocontractile myocardial tissue determines postinfarction left ventricle remodeling. The aim of this pilot study was to evaluate the revascularization effect in a group of patients with ischemic cardiomyopathy and LV systolic dysfunction indicated for surgical revascularization, based on evidence for multivessel disease on coronarography and viable myocardium (CMR, SPECT).

Aims: To evaluate the revascularization effect in patients with ischemic LV systolic dysfunction and to find preoperative predictors of revascularization effect.

Methods: 33 patients (64 \pm 11 years) with baseline LVEF $34.9 \pm 9.3 \%$ were included in the study. After a follow-up of $10.7 \pm 1.2$ months, ECHO and SPECT were performed again. The whole group of patients was divided according to revascularization effect ( $\uparrow$ LVEF $>5 \%$ and $\downarrow$ LVESV $>5 \%$ compared with baseline) into revascularization responders $(\mathrm{R}, \mathrm{n}=22)$ and nonresponders $(\mathrm{NR}, \mathrm{n}=11)$.

Results: At baseline there was no difference between the subgroups in LVEF ( $R=35.7 \pm 11.0 \%$ vs. $N R=34.3 \pm 8.2 \%)$, $\operatorname{EDV}(\mathrm{R}=183.6 \pm 43.2$ vs. $\mathrm{NR}=180.2 \pm 80.5 \mathrm{ml}), \mathrm{ESV}(\mathrm{R}=118.5 \pm 40.4$ vs. $\mathrm{NR}=119.7 \pm 55.2 \mathrm{ml})$.

The responders showed in a revascularization effect subanalysis differences in the values of LVEF $(+9.8 \pm 8.1 \%$, $\mathrm{p}<0.009)$, reduction of $\operatorname{EDV}(-39.9 \pm 50.9 \mathrm{ml}, \mathrm{p}=0.05)$ and $\operatorname{ESV}(-35.4 \pm 42.6 \mathrm{ml}, \mathrm{p}=0,002)$ compared with baseline.

The only preoperative parameters predicting $L V$ reverse remodeling were the $T_{E-E m}(R=-10.6 \pm 44.1$ vs. $N R=$ $29.7 \pm 43.7 \mathrm{~ms}, \mathrm{p}=0.037)$ and the size of fixed perfusion defect (FPD) $(\mathrm{R}=11.9 \pm 13.5 \mathrm{vs}$. $\mathrm{NR}=22.9 \pm 15.3 \%$ of $\mathrm{LV}$, $\mathrm{p}=0.044)$.

Conclusions: Patients with ischemic LV systolic dysfunction with a preoperatively determined myocardial viability develop LV reverse remodeling. The only preoperative parameters predicting LV reverse remodeling were echocardiographic $\mathrm{T}_{\mathrm{E}-\mathrm{Em}}$ and FPD on SPECT.

\section{INTRODUCTION}

Chronic heart failure is a clinical syndrome that in United States alone affects about 5 million patients per year. It is responsible for approximately 1,000,000 hospitalizations and 300,000 deaths ${ }^{1 .}$ The most common cause of chronic left-sided heart failure in developed countries is ischemic heart disease (IHD) (ref., ${ }^{2,3}$ ).

One prognostic factor emerging from a number of laboratories, clinical and functional tests, is the ejection fraction of the left ventricle (LVEF) (ref. $\left.{ }^{4}\right)$. Endsystolic and enddiastolic LV volumes are the basic measured parameters from which LVEF can be assessed using a simple calculation. A single evaluation of enddiastolic or endsystolic volume produces quantitative information about the level of LV remodeling/ the possibility of its reversibility after the start of particular myocardial treatment. LV volume assessment also provides important prognostic information after myocardial infarction and after surgical correction for valvular diseases ${ }^{5-7}$.

IHD plays a role in triggering heart failure by a number of mechanisms. These can be divided into two groups according to possible therapeutic intervention. The first group of probably irreversible processes includes primarily myocardial necrosis with subsequent development of scar leading to $\mathrm{LV}$ remodeling which depends on the extent of the infarction. As potentially curable causes of heart failure, can be considered, stunning and hibernation of myocardium which represent a functional adaptation state to acute or chronic myocardial ischemia ${ }^{8}$.

Currently, there are available a number of imaging methods with high diagnostic accuracy for revealing the presence of viable myocardium or nonviable scar. Routinely used radionuclide methods such as single photon emission tomography (SPECT) using perfusion radiopharmacs $\left({ }^{201}\right.$ thalium, ${ }^{99 \mathrm{~m}}$ technecium-MIBI) and positron 
emission tomography (PET) with a metabolic radiopharmaceutical $\left({ }^{18} \mathrm{~F}\right.$-deoxyglucose $)$ have higher sensitivity and slightly lower specificity in viable myocardium detection than stress echocardiography ${ }^{8}$.

The correct selection of patients for surgery with an extensive hibernating myocardium mass before revascularization is pivotal from the viewpoint of assessment of revascularization effects apropos the relatively high periprocedural risk attending the surgical revascularization of the myocardium. The problem is the coexistence of scar and other individual ischemic substrates (hibernation and stunning respectively) that can be, to varying extent present in the same patient. Another no less important factor is the duration of LV remodeling and its severity. Presently there are limited data on predictors of revascularization in terms of reverse remodeling onset and systolic and diastolic function improvement as pivotal markers of the prognostic benefits to revascularized patients. The amount of viable myocardium or the ratio of hibernating healthy myocardium and nonviable myocardium needed for optimal revascularization so that the outcome of revascularization outweighs its risk is not definitely known. What level of LV remodeling is an irreversible phase of cardiomyopathy remains another question?.

The aim of this pilot study was to evaluate the revascularization effect in a group of patients with ischemic cardiomyopathy and LV systolic dysfunction indicated for surgical revascularization, based on evidence of multivessel disease on coronarography and viable myocardium. Another aim was to find pre-operative predictors of revascularization effects in terms of LV reverse remodeling induction.

\section{Patient group}

A total of 33 consecutive patients aged $63.7 \pm 11.7$ years were included in the study. All were examined within the frame of prospective follow-up of patients with ischemic cardiomyopathy based on coronarography and viability of myocardium from other examinations (cardiac gated SPECT, cardiac magnetic resonance imaging) for surgical revascularization of myocardium. Patients with acute coronary syndrome in the preceding three months were excluded.

The basic clinical characteristics and results of evaluated parameters for the whole group and subgroups of responders and nonresponders of revascularization at baseline are depicted in Table 1.

After an average length of follow-up of $10.7 \pm 1.2$ months after surgery and $11.6 \pm 1$ month after preoperative selection, all patients were regularly examined clinically, echocardiographically and by cardiac gated-SPECT. The whole patient group $(\mathrm{n}=33)$ was then divided into 2 subgroups: revascularization responders $(n=22)$ and revascularization nonresponders $(n=11)$. The selection was made according to the onset of reverse remodeling assessed from results of the control cardiac gated-SPECT (as higher SPECT reproducibility of selected parameters compared with echocardiography), whilst the criterion for inclusion in the responder group was a postoperative increase by $>5 \%$ and reduction of LVESV $>5 \%$ compared to preoperative baseline values of the given patient ${ }^{9}$.

All patients had optimized treatment for ischemic heart disease and heart failure comparable for responders and nonresponders; all were in the NYHA class I-III. All patients were part of a complex LV myocardium viability and function assessment and after signing written consent examined echocardiographically, by cardiac gated-SPECT. All examinations were performed in tight sequence during one day in one center and were evaluated double-blind.

\section{Echocardiography}

Echocardiographic examinations were performed on GE Ultrasound Vivid 7 (GE Healthcare Technologies, Waukesha, Wisconsin, USA) equipped with a multifrequency ultrasound probe. The evaluation of echocardiographic findings was performed off-line in an environment of archival program EchoPAC 7 Option (version BT 06) blindly without knowledge of clinical status or results of other examinations. The resting examination with synchronous registration of 1 ECG lead was performed standardly from parasternal projections on the long and short axis of LV and from apical 2, 3 and 4 chamber projections. Endsystolic and enddiastolic volumes were assessed from 2-dimensional images (B-mode) - endsysto-

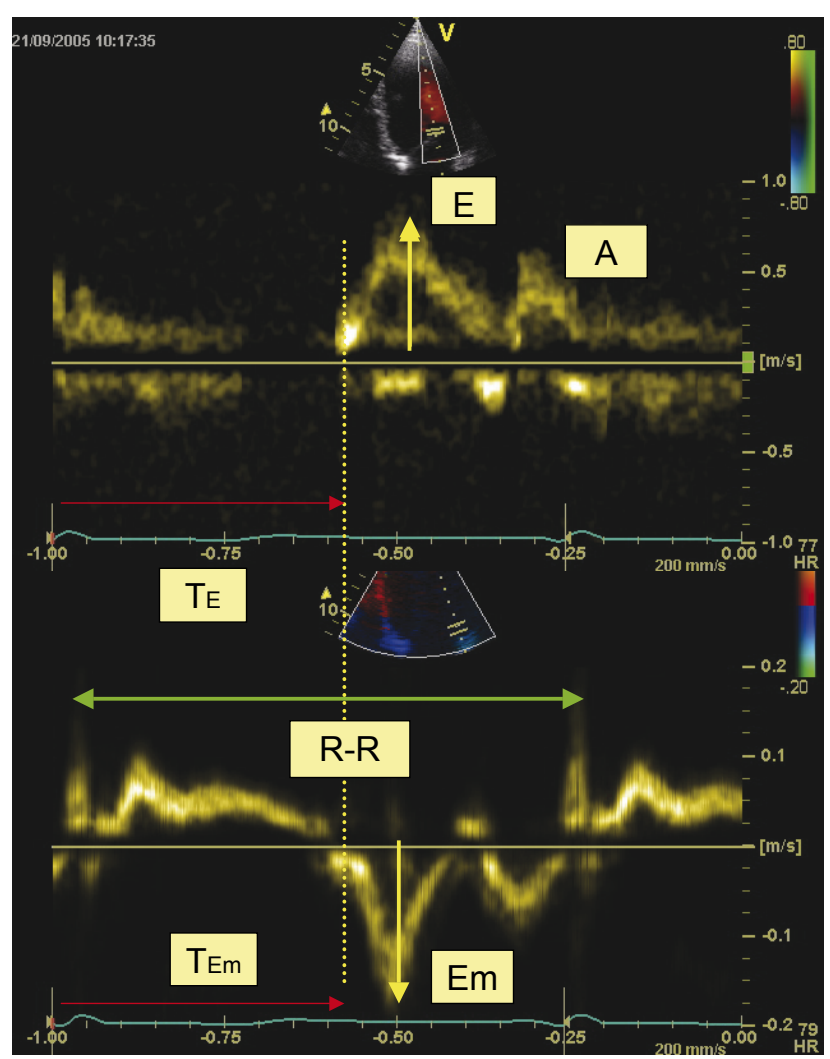

Fig. 1. Measured echocardiographic parameters from PWD of transmitral flow (upper part) and PWTDE of mitral annulus (lower part). (Early diastolic transmitral flow velocity $\mathrm{E}$, diastolic mitral annular velocities Em, time interval between onset of early transmitral flow and early annular mitral motion $\mathrm{T}_{\mathrm{E}-\mathrm{Em}}$ ). 
Table 1. Baseline clinical characteristics of the whole group and revascularization responders and nonresponders subgroups.

\begin{tabular}{|l|c|c|c|}
\hline & $\begin{array}{c}\text { whole group } \\
(\mathrm{n}=33)\end{array}$ & $\begin{array}{c}\text { nonresponders } \\
(\mathrm{n}=11)\end{array}$ & $\begin{array}{c}\text { responders } \\
(\mathrm{n}=22)\end{array}$ \\
\hline Men/women & $(21 / 12)$ & $(7 / 4)$ & $(14 / 8)$ \\
\hline Mean age (yr) & $63.7 \pm 11.7$ & $62.6 \pm 12$ & $64.1 \pm 11.2$ \\
\hline Myocardial infarction in history (n) & $28(85 \%)$ & $11(100 \%)$ & $17(78 \%)$ \\
\hline Chest pain - AP (CCS class) & $1.2 \pm 1.27$ & $1.22 \pm 1.3$ & $1.04 \pm 1.1$ \\
\hline NYHA (class) & $2.2 \pm 1.8$ & $2.3 \pm 1.9$ & $2.13 \pm 2$ \\
\hline Type 2 DM (n) & $13(39 \%)$ & $6(54 \%)$ & $7(32 \%)$ \\
\hline Hypertension (n) & $24(73 \%)$ & $11(100 \%)$ & $13(59 \%)$ \\
\hline Stroke in history (n) & $6(18 \%)$ & $3(27 \%)$ & $3(14 \%)$ \\
\hline Smokers (n) & $14(42 \%)$ & $3(27 \%)$ & $11(55 \%)$ \\
\hline Atrial fibrillation $(\mathrm{n})$ & $7(21 \%)$ & $3(27 \%)$ & $4(18 \%)$ \\
\hline 2-vessel disease $(\mathrm{n})$ & $12(36 \%)$ & $4(36 \%)$ & $8(36 \%)$ \\
\hline 3-vessel disease $(\mathrm{n})$ & $21(64 \%)$ & $7(63 \%)$ & $14(63 \%)$ \\
\hline LMCA disease $(\mathrm{n})$ & $10(30 \%)$ & $5(50 \%)$ & $5(23 \%)$ \\
\hline LAD disease $(\mathrm{n})$ & $31(94 \%)$ & $10(91 \%)$ & $21(95 \%)$ \\
\hline Bypass number $(\mathrm{n})$ & $3.3 \pm 0.92$ & $3.3 \pm 0.82$ & $3.28 \pm 0.96$ \\
\hline
\end{tabular}

$\mathrm{p}=\mathrm{NS}$ (responders vs. nonresponders in individual parameters)

Table 2. Baseline values of evaluated echocardiographic and gated-SPECT parameters of the whole group, revascularization responders and nonresponders subgroups before surgery.

\begin{tabular}{|c|c|c|c|}
\hline & $\begin{array}{c}\text { whole group } \\
(\mathrm{n}=33)\end{array}$ & $\begin{array}{c}\text { nonresponders } \\
(\mathrm{n}=11)\end{array}$ & $\begin{array}{c}\text { responders } \\
(\mathrm{n}=22)\end{array}$ \\
\hline LVEF ECHO biplane (\%) & $34.9 \pm 9.3$ & $35.7 \pm 11$ & $34.3 \pm 8.2$ \\
\hline LVEF gated SPECT (\%) & $34.7 \pm 9.4$ & $34.9 \pm 11.1$ & $34.6 \pm 8.4$ \\
\hline EDV ECHO biplane $(\mathrm{ml})$ & $181.6 \pm 66.3$ & $183.5 \pm 43.2$ & $180.2 \pm 80.5$ \\
\hline EDV gated SPECT (ml) & $204.9 \pm 71.7$ & $198.5 \pm 60.4$ & $208.2 \pm 78.2$ \\
\hline ESV ECHO biplane $(\mathrm{ml})$ & $119 \pm 56.2$ & $118.5 \pm 40.4$ & $120.6 \pm 65.3$ \\
\hline ESV gated SPECT $(\mathrm{ml})$ & $138.1 \pm 62.6$ & $133.5 \pm 56.9$ & $140.6 \pm 66.6$ \\
\hline Total perfusion defect- PD (\% LV) & $37.1 \pm 16.5$ & $39.5 \pm 18.9$ & $38 \pm 35.8$ \\
\hline Fixed perf. defect- FPD (\% LV) & $15.7 \pm 14.9$ & $22.9 \pm 15.3$ & $11.9 \pm 13.5 *$ \\
\hline PD-FPD (\% LV) & $21.2 \pm 13.2$ & $16.6 \pm 12.5$ & $23.5 \pm 13.3$ \\
\hline $\mathrm{PD} / \mathrm{FPD}$ ratio & $2.36 \pm 1.89$ & $1.72 \pm 1.3$ & $3.2 \pm 2.1^{*}$ \\
\hline $\mathrm{E}(\mathrm{cm} / \mathrm{s})$ & $76.9 \pm 25.1$ & $71.4 \pm 25.2$ & $80.8 \pm 25.2$ \\
\hline $\mathrm{A}(\mathrm{cm} / \mathrm{s})$ & $67.5 \pm 28.7$ & $67.8 \pm 30.2$ & $67.3 \pm 28.9$ \\
\hline $\mathrm{E} / \mathrm{A}$ & $1.4 \pm 0.9$ & $1.3 \pm 0.8$ & $1.5 \pm 1$ \\
\hline $\mathrm{Sm}(\mathrm{cm} / \mathrm{s})$ & $3.4 \pm 1.2$ & $3.3 \pm 1.2$ & $3.5 \pm 1.3$ \\
\hline $\operatorname{Em}(\mathrm{cm} / \mathrm{s})$ & $3.4 \pm 1.1$ & $3.3 \pm 1.4$ & $3.4 \pm 0.8$ \\
\hline $\mathrm{T}_{\mathrm{E}-\mathrm{Em}}(\mathrm{ms})$ & $6.2 \pm 47.4$ & $29.7 \pm 43.6$ & $-10.6 \pm 44.1^{*}$ \\
\hline
\end{tabular}

$* \mathrm{p}<0.05$ (responder vs. nonresponders)

(E - early transmitral flow velocity, A - late transmitral flow velocity, E/A ratio, Sm - average systolic mitral annular velocity, Em - average early diastolic mitral annular velocity, $\mathrm{T}_{\mathrm{E}-\mathrm{Em}}$ - time interval between onset of early transmitral flow and early annular mitral motion) 
lic and enddiastolic planimetry of endocardial border of LV were performed in the undermentioned echocardiographic projections ${ }^{10}$. LV volumes and EF were assessed using Simpson's disc method from 2 apical projections on LV long axis, apical 4-chamber projection first followed by apical 2-chamber projection. All parameters were assessed as averages of 3 measures of 1-cycle recordings during stable sinus rhythm. In case of atrial fibrillation $(n=7$, $21 \%$ of patients) the particular parameters were obtained by averaging from heart cycles with identical R-R intervals (differences between evaluated R-R intervals $<5 \mathrm{~ms}$ ). Two investigators without knowledge of clinical, gated SPECT, MRI and angiographic data, performed off-line analysis of the echocardiograms. Disagreements in interpretation were resolved by consensus.

The measured echocardiographic parameters were LV volumes and LVEF evaluated using biplanar echocardiographic approach (apical 2-chamber and apical 4-chamber projections), transmitral flow velocities E, A, E/A ratio, systolic mitral annular velocity $\mathrm{Sm}$, diastolic mitral annular velocities Em, Am, time interval between onset of early transmitral flow and early annular mitral motion $\mathrm{T}_{\mathrm{E}-\mathrm{Em}}$, which was chosen in a post-hoc analysis. (Fig. 1)

\section{Gated technetium-99m sestamibi (MIBI)} single photon emission computed tomography

\section{Rest Imaging after Nitrate Administration}

All patients underwent 1-day rest imaging with nitrate administration: $8 \mathrm{mCi}(296 \mathrm{MBq}){ }^{99 \mathrm{~m}}$ technecium-MIBI was injected at rest, with SPECT imaging performed 1 hour after injection. Three hours later, $24 \mathrm{mCi}(888 \mathrm{MBq})$ ${ }^{99 \mathrm{~m}}$ technecium-MIBI was injected after sublingual nitrate administration 1 hour before SPECT imaging.

\section{Scintigraphy}

Gated SPECT imaging ( 64 projections from the $45^{\circ}$ right anterior oblique projection to the $45^{\circ}$ left posterior oblique projection, 8 frames/cycle) was performed using a 2-detector gamma camera (ecam, Siemens, Erlangen, Germany) equipped with a low-energy, high-resolution parallel-hole collimators. Myocardial perfusion images were analyzed visually and quantitatively on computergenerated polar maps using automated, commercially available software 4D-MSPECT package (University of Michigan, Ann Arbor, MI, USA). Gated single photon emission computed tomography rest left ventricular ejection fractions (LVEF) and left ventricular enddiastolic/ endsystolic volumes (EDV/ESV) were obtained using software 4D-MSPECT.

For further analysis, the values of resting cardiac gated-SPECT were used, i.e. resting perfusion defect size (deterioration of $2.5 \mathrm{SD}$ compared to normal database, expressed as \% of total LV), nonviable myocardium (fixed perfusion defect with an accumulation below $50 \%$ of maximum, expressed as \% of total LV) and resting values of LVEF, EDV and ESV.

\section{Statistical analysis}

Statistical analysis was performed on statistical software SPSS for Windows 12.0 (SPSS Inc., Chicago, USA).
Paired t-tests for dependent samples were used for statistical evaluation of differences between individual subgroups at baseline and after revascularization of myocardium. Receiver operating curve (ROC) analysis was used for assessment of sensitivity and specificity of individual parameters for presurgical prediction of LV reverse remodeling. $\mathrm{p} \leq 0.05$ was the chosen level of significance.

\section{RESULTS}

\section{Characteristics of the whole group, subgroup of responders and nonresponders of revascularization at baseline.}

Baseline values of evaluated echocardiographic and gated-SPECT parameters of the whole group, revascularization responders and nonresponders subgroups before surgery are depicted in Table 2.

The differences in the measurements of LVEF between biplane echocardiography and cardiac gated SPECT were not statistically significant. However from the statistics there was a significant difference between enddiastolic and endsystolic volumes that were systematically underestimated by echocardiography. The values from the gated-SPECT of myocardium were reliable and accurate 10.7 months after surgery in terms of the ability to divide the whole group into responders and nonresponders subgroups.

From the statistical analysis of revascularization responder and nonresponder subgroups differences existed in the number of patients with fixed perfusion defect (responders $61 \%$ vs. nonresponders $47 \%$ ). The presence of a nonviable myocardium (fixed perfusion defect) in each particular coronary artery area was no different in the areas of left anterior descending artery anterior, circumflex artery or right coronary artery. There were no differences in localization of hemodynamically significant coronary artery stenoses, completeness of surgical revascularization (responders $80 \%$ vs. $61 \%$ nonresponders).

\section{Influence of cardiovascular revascularization on monitored parameters of the whole group and subgroups of responders and nonresponders of revascularization.}

After an average duration of $10.7 \pm 1.2$ months after surgical revascularization, the echocardiography and gated-SPECT of myocardium were checked.

The influence of surgical revascularization on LVEF (\%), ESV (ml) and EDV (ml) in the whole group, revascularization responder and nonresponder subgroups after surgery evaluated by echocardiography and cardiac gatedSPECT are depicted in Figures 2, 3, 4.

For other examined parameters in the whole group there was a significant reduction in resting perfusion defect from baseline values of $37.1 \pm 16.5$ to $30.2 \pm 22.5 \%$ of $\mathrm{LV}, \mathrm{p}=0.029$. In the responder subgroup the value of total perfusion defect decreased after revascularization from baseline values of $35.8 \pm 15.5$ to $23.8 \pm 22 \%$ of $L V, p \leq 0.05$. In the nonresponder subgroup, the total perfusion defect remained stable after revascularization with the entry values of $39.5 \pm 18.9$ and control values of $42.4 \pm 18.6 \%$ of $\mathrm{LV}$, $\mathrm{p} \leq \mathrm{NS}$. Values of the other parameters (fixed perfusion 

remodeling after surgical revascularization in patients with ischemic cardiomyopathy and left ventricle systolic dysfunction

defect, E, A, E/A, Sm, Em, $\mathrm{T}_{\mathrm{E}-\mathrm{Em}}$ ) remained unchanged after surgical revascularization

\section{Preoperative predictors of revascularization in relation to reverse remodeling onset.}

In a retrospective analysis of revascularization of myocardium responder and nonresponder subgroups in terms of a possible preoperative prediction of myocardial
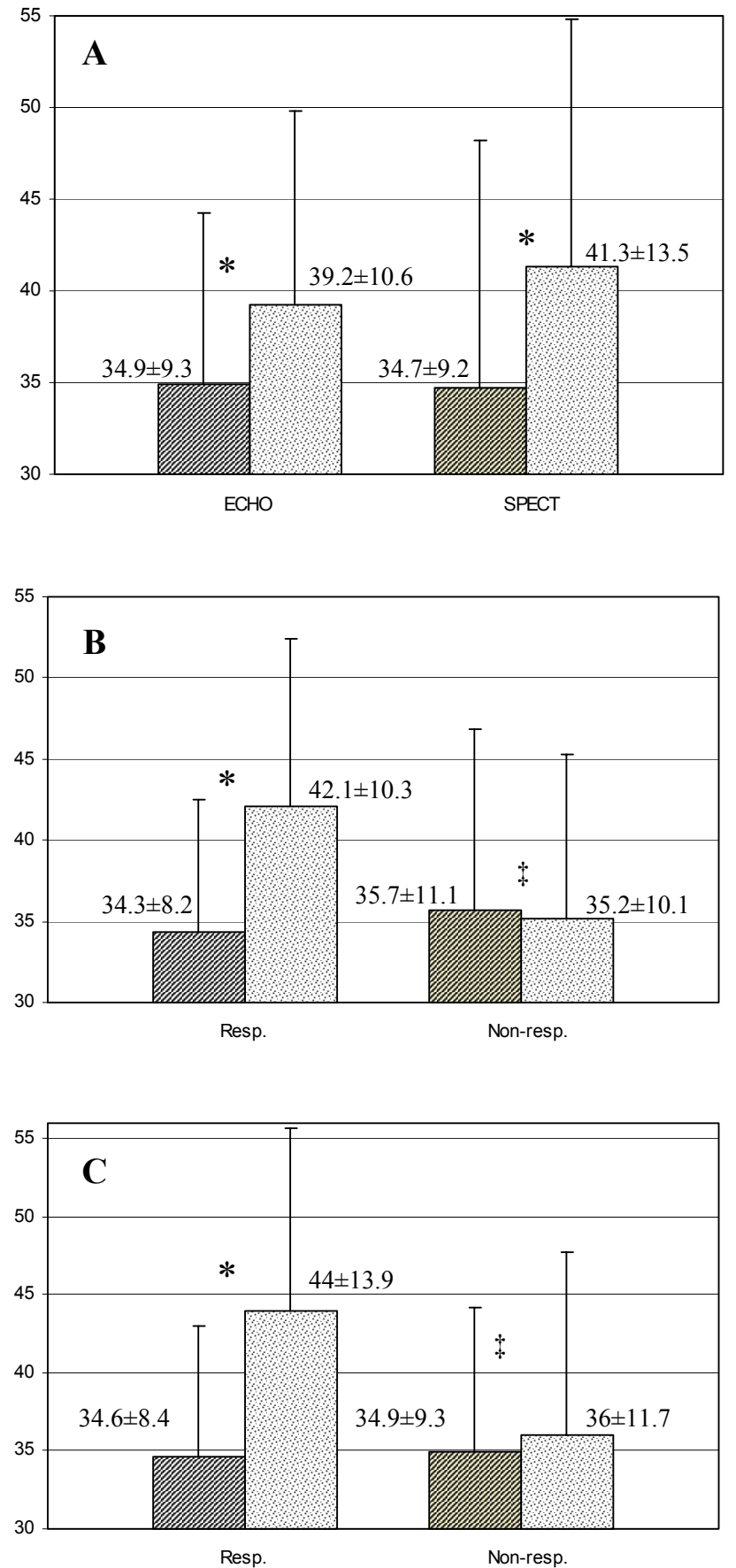

Fig. 2. Influence of surgical revascularization on LVEF (\%) in the whole group (A), revascularization responders and nonresponders subgroups 10.7 months after surgery evaluated by echocardiography (B) and cardiac gated-SPECT (C). $\left({ }^{*} \mathrm{p} \leq 0.05,{ }^{\star} \mathrm{p}=\mathrm{NS}\right)$. revascularization, the result apropos LV reverse remodeling onset (post-operative increase of LVEF $\geq 5 \%$ and a simultaneous decrease of LV endsystolic volume $\geq 5 \%$ compared to baseline values) statistically significant differences were found in two examined parameters. The first parameter in which both subgroups had a preoperative difference was the range of a fixed perfusion defect measured by gated-SPECT of myocardium (responders $11.9 \pm 13.5 \%$
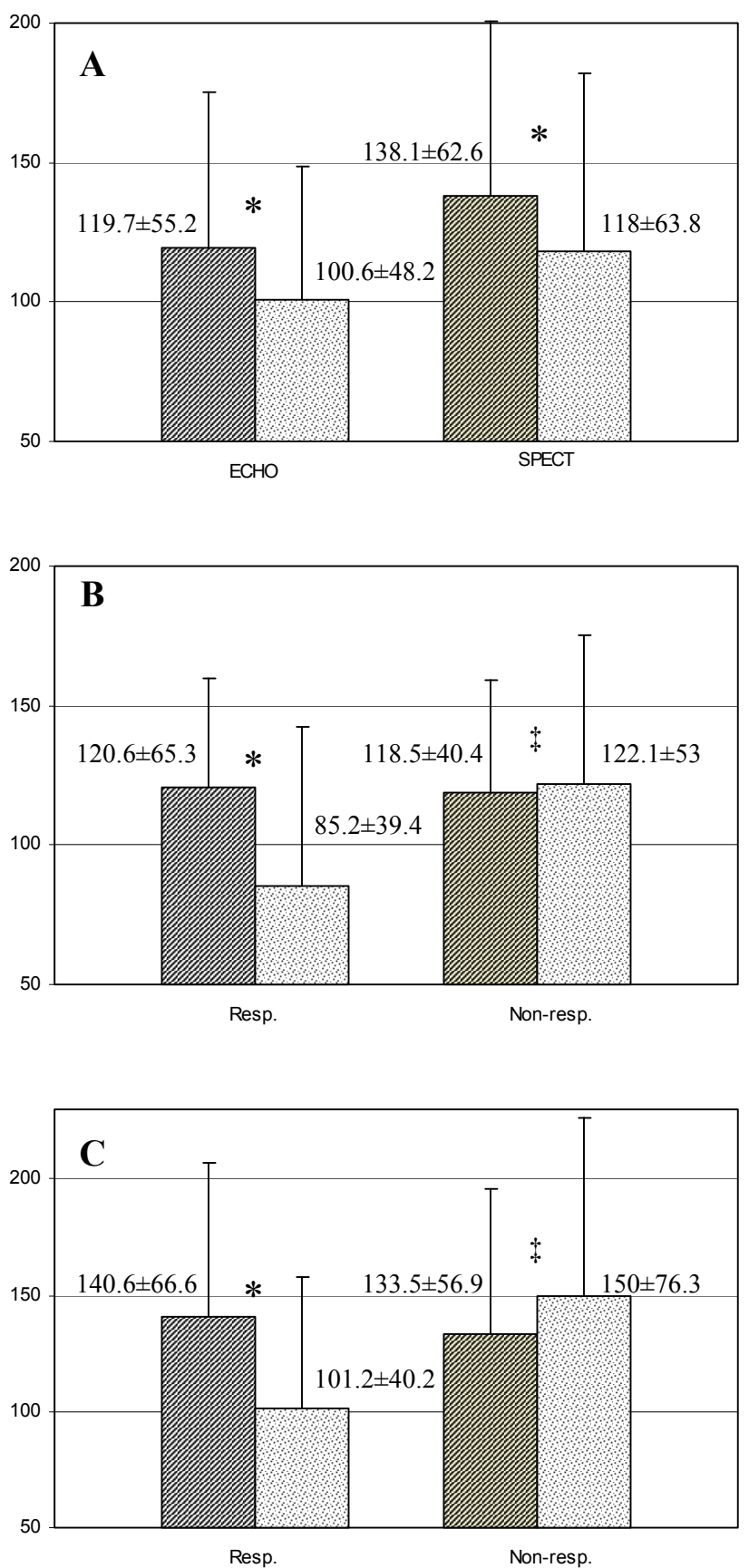

Fig. 3. Influence of surgical revascularization on ESV of $\mathrm{LV}(\mathrm{ml})$ in the whole group (A), revascularization responders and nonresponders subgroups 10.7 months after surgery evaluated by echocardiography (B) and cardiac gated SPECT (C). $\left({ }^{*} p \leq 0.05\right.$, ${ }^{\ddagger} \mathrm{p}=\mathrm{NS}$ ). 
$\mathrm{LV}$ vs. nonresponders $22.9 \pm 15.3, \mathrm{p}=0.044)$. Cut-off value $<15 \%$ of LV predicts LV reverse remodeling with sensitivity of $72 \%$ and specificity of $73 \%$. (Fig. 5)

Another parameter in which both subgroups differed significantly was an entry value of echocardiographic parameter $\mathrm{T}_{\mathrm{E}-\mathrm{Em}}$, which was significantly different in the responder subgroup (-10.6 \pm 44.1 compared to $29.7 \pm 43.7$ $\mathrm{ms}$ in nonresponder subgroup, $\mathrm{p}=0.037$ ).
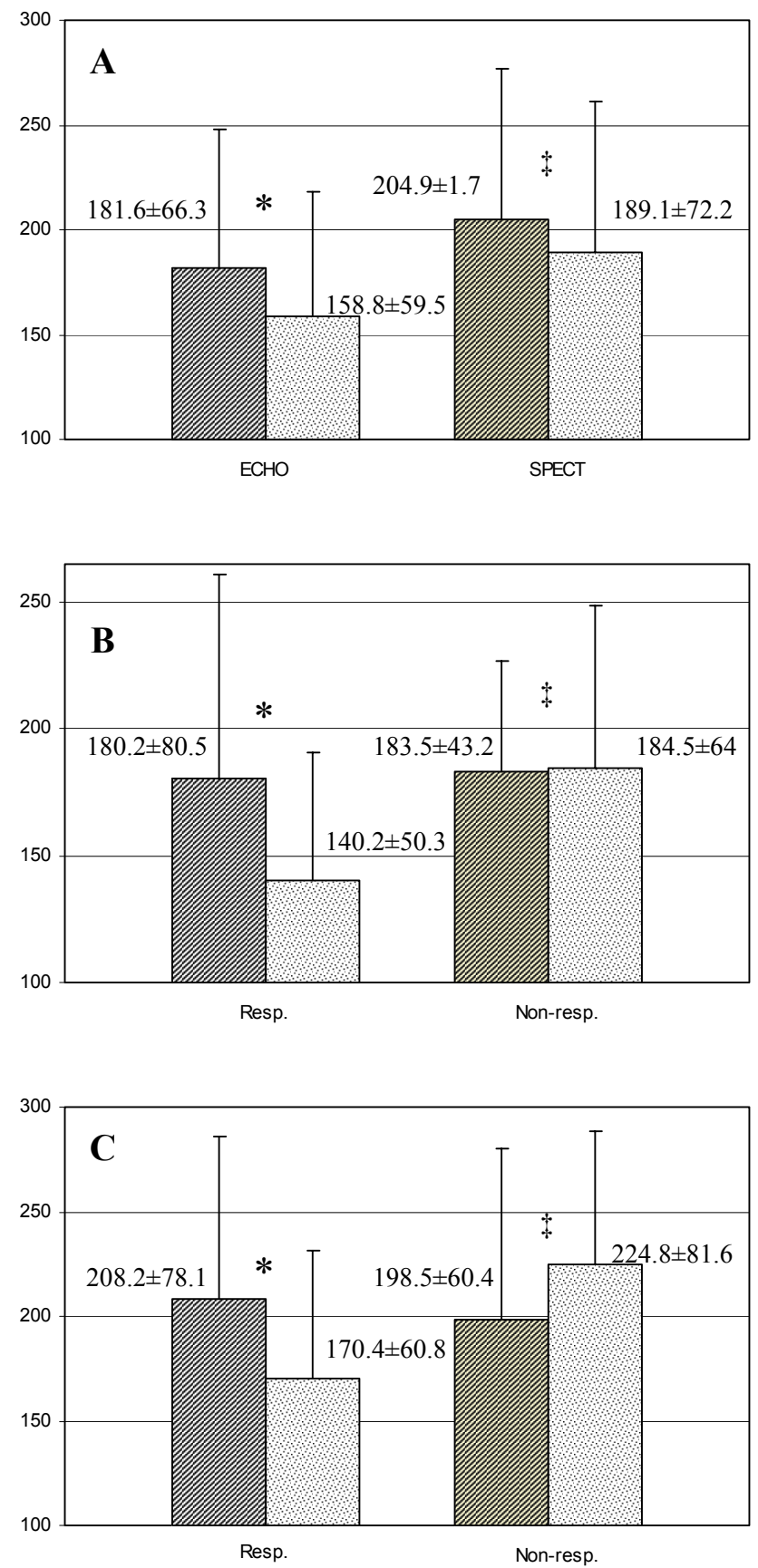

Fig. 4. Influence of surgical revascularization on EDV of $\mathrm{LV}(\mathrm{ml})$ in the whole group (A), revascularization responders and nonresponders subgroups 10.7 months after surgery evaluated by echocardiography (B) and cardiac gated SPECT (C). ( ${ }^{*} \mathrm{p} \leq 0.05$, ${ }^{\mathrm{p}}=\mathrm{NS}$ ).
Receiver operating curve (ROC) showed that a cut-off value of $T_{E-E m}<13 \mathrm{~ms}$ predicts reverse remodeling of $\mathrm{LV}$ after revascularization with a sensitivity $78 \%$ and specificity $78 \%$. (Fig. 6)

Pre-operative results of other examined parameters (LVEF, EDV, ESV, E, A, E/A, Em, total perfusion defect) were not significantly different.

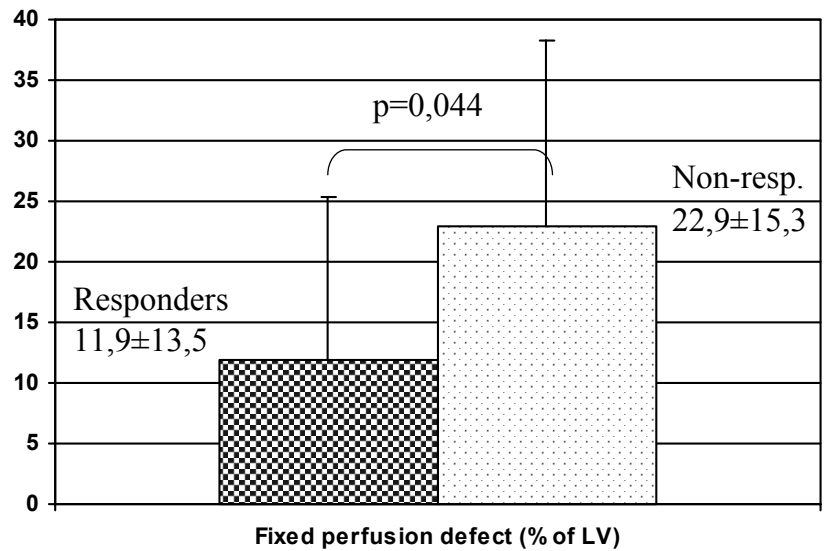

Fig. 5. Preoperative values of fixed perfusion defect assessed by cardiac gated SPECT (responders $11.9 \pm 13.5 \% \mathrm{LV}$ vs. nonresponders $22.9 \pm 15.3$, $\mathrm{p}=0.044)$. Cut-off $<15 \%$ of LV in ROC analysis predicts LV reverse remodeling with sensitivity of $72 \%$ and specificity of $73 \%$.

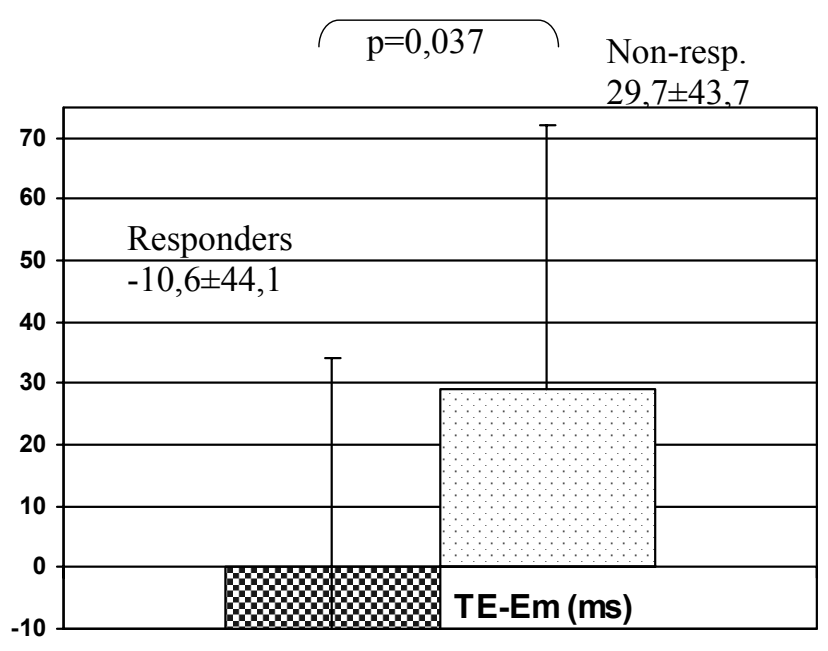

Fig. 6. Preoperative values of $T_{E-E m}$ by echocardiography with a statistically significant difference in the responders subgroup $-10.6 \pm 44.1$ compared to nonresponders subgroup $29.7 \pm 43.7 \mathrm{~ms}, \mathrm{p}=$ 0.037 . Receiver operating curve (ROC) showed that a cut-off value of $\mathrm{T}_{\mathrm{E}-\mathrm{Em}}<13 \mathrm{~ms}$ predicts $\mathrm{LV}$ reverse remodeling after revascularization with a sensitivity $78 \%$ and specificity $78 \%$. 


\section{DISCUSSION}

Revascularization of myocardium represents a way of treating patients with ischemic cardiomyopathy mainly with systolic LV dysfunction. Patients with preoperative evidence of a significant mass of viable myocardium and multivessel disease have significant benefit from this procedure $^{11}$. A relatively high periprocedural risk needs to be considered in patients with systolic LV dysfunction. Perioperative mortality of aorto-coronary bypass surgery in this specific group ranges according to several reports from approximately $5 \%$ in younger adults and up to $30 \%$ in older adults with more severe LV systolic dysfunction and depending on its severity, age, sex, surgery urgency, severity of coronary arteries affection, complicating factors, co-morbidities and other factors 8 .

A preoperative finding of a significant mass of hibernating myocardium before surgery is fundamental for clinical management and selection of patients for revascularization. The outcome of revascularization can be significantly affected by coexistence of a scar tissue and individual ischemic substrates (hibernation, stunning) in one patient, severity and length of lasting of LV remodeling. It is necessary to emphasize that the aim of revascularization is not only an amelioration of the symptoms of heart failure but also increased survival, onset of LV reverse remodeling and prevention of its progression caused by a deterioration of LV systolic and diastolic function and prevention of myocardium infarction and sudden cardiac death rate by elimination of arrhythmogenic substrates ${ }^{8}$ Considering the revascularization of myocardium effect in our study there was an evident postoperative reduction of LV volumes and increase in LVEF after revascularization in the whole patient group but mainly in the revascularization responder subgroup. This finding is in a good agreement with a number of other studies published in this area ${ }^{8,9,11,13,14}$. A postoperative statistically significant reduction of total perfusion defect extent on SPECT and a stationary extent of a fixed perfusion defect representing nonviable myocardial scar tissue was also evident. We found a statistically significant difference in extent of nonviable myocardium by SPECT at baseline between the responders and nonresponders of the revascularization subgroups. Presently there are limited data on predictors of revascularization in terms of reverse remodeling onset and systolic and diastolic function improvement, which are considered to be the fundamental markers of prognostic benefit of revascularized patients with proven hibernating myocardium. Regression of heart failure symptoms, improvement in exercise capacity and a level of LV reverse remodeling directly in the majority of studies correlated with the preoperative extent of a presumably viable myocardium. Lombardo et al. found that a preoperative documentation of viable myocardium of at least $13 \%$ of LV by dobutamine echocardiography is needed for a reverse LV remodeling from 36 to $41 \%{ }^{12}$. Ragosta et al. showed that a preoperative extent of viable myocardium of at least $53 \%$ assessed using ${ }^{201}$ thalium-SPECT is needed for a statistically significant postoperative increase in LV from
$29 \%$ to $41 \%{ }^{13}$. According to various studies using mostly radionuclide methods, this value ranges from 8 to $67 \%$ of $\mathrm{LV}^{8,14}$. The extent of viable myocardium assessed using gate-SPECT of myocardium ranging from 25 to $30 \%$ of $\mathrm{LV}$ can be considered a reasonable cut-off value for a relatively accurate prediction of reverse $\mathrm{LV}$ remodeling onset (postoperative increase of LVEF $>5 \%$ ) assessed using receiver operating curve (ROC) analysis9.

To date, there is one study in which patients with substantial nonviable myocardium on ${ }^{18} \mathrm{~F}-\mathrm{FDG} / \mathrm{SPECT}$ are predicted to have a poor early functional outcome ${ }^{26}$. Based on this finding we tested a fixed perfusion defect parameter assessed using gated-SPECT of myocardium as a marker of a nonviable myocardial scar tissue in the light of reverse LV remodeling prediction defined as a simultaneous increase of LVEF of at least $5 \%$ and decrease of EDV of at least $5 \%$ evaluated after 11 months from revascularization, which we consider a long enough time interval for an onset of a functional recovery of hibernating regions of myocardium after coronary revascularization. Gated-SPECT of myocardium was considered a reference method for evaluation of $\mathrm{LV}$ reverse remodeling. It is a clinically robust method, highly reproducible and relatively independent of the examiner. In the responder subgroup we reported an LVEF increase of $9.8 \%$, EDV reduction of $40 \mathrm{ml}$ and $\mathrm{ESV}$ reduction of $35.4 \mathrm{ml}$ compared to baseline values. The only statistically significant gatedSPECT predictor of revascularization result in terms of reverse remodeling onset in our study was surprisingly not baseline preoperative value of total extent of perfusion defect but a value of fixed perfusion defect (infarct size). This parameter can be considered a marker of scar tissue after myocardial infarction. There is a close association between fibrosis in human hearts and infarct size assessed by sestamibi gated SPECT. Sestamibi infarct size has been quantified using a standardized approach based on cardiac phantom studies ${ }^{25}$. The presence and extent of nonviable myocardium is a very strong mortality predictor in patients with ischemic heart disease ${ }^{15}$. Cut-off values of this parameter (fixed perfusion defect) below $15 \%$ of LV had in our study a relatively high sensitivity and specificity in $\mathrm{LV}$ reverse remodeling after revascularization. We can presume that for a reverse $\mathrm{LV}$ remodeling it is necessary to show a preoperative mass of viable myocardium of at least $85 \%$ of LV. It is a relatively higher value than in available studies using radionuclide methods in patients with LV systolic dysfunction ${ }^{8,13,14}$. However, for this result we needed to take into account preoperative LV systolic function, rate of LV remodeling and patient's co-morbidities. All patients in the revascularization nonresponder subgroup had a history of myocardial infarction compared to only $71 \%$ of patients after myocardial infarction in the responder subgroup. This difference was not statistically significant. Another fact that has to be mentioned is a larger number of patients with a nonviable myocardium in revascularization responders subgroup compared to nonresponders subgroup (61 vs. $41 \%$ ). That means that not every patient after myocardial infarction had an evident fixed perfusion defect on gated-SPECT of myocardium. The above-men- 
tioned number of patients with a nonviable myocardium is nevertheless larger than for instance the patient group of Hoilund-Carlsen et al. ( $8 \%$ of 168 revascularized patients with a stable angina pectoris $)^{16}$. This fact is in our study caused by a selection of patients with a systolic LV dysfunction with a large number of patients after myocardial infarction. An absolute value of fixed perfusion defect in our patient group ( $12 \%$ of LV in responders vs. $23 \%$ in nonresponders) demonstrates a larger extent of nonviable myocardial tissue in the revascularization nonresponders subgroup. We found no correlation between localization of nonviable myocardium in individual coronary artery perfusion areas and revascularization outcome. Thus, the only preoperative predictor of revascularization in terms of reverse LV remodeling onset in patients with ischemic cardiomyopathy is extent of nonviable myocardium.

We found a statistically significant difference in values of $\mathrm{T}_{\mathrm{E}-\mathrm{Em}}$ between revascularization responders and nonresponders subgroups. Patients in the responder subgroup had a negative value of $\mathrm{T}_{\mathrm{E}-\mathrm{Em}}$. This means that an average annular motion velocity $\mathrm{T}_{\mathrm{E}}$ lags behind the onset of transmitral flow E wave. Patients in the nonresponder subgroup had positive values of $\mathrm{T}_{\mathrm{E}-\mathrm{Em} \text {. }}$ That means that the early annular motion velocities preceded onset of transmitral flow. The lag of onset of early annular mitral motion behind the beginning of diastolic LV filling was for the first time described by Garcia et al. in patients with restrictive cardiomyopathy, whilst in a group of healthy control subjects Em preceded E wave ${ }^{17}$. Hasegawa et al. found in another experimental work a relationship between time interval $\mathrm{T}_{\mathrm{E}-\mathrm{Em}}$ and diastolic relaxation constant tau assessed using catheterization a progressive decrease of peak mitral annular velocity Em and its lag behind the beginning of wave E of transmitral flow ${ }^{18}$. Rivas-Gotz et al. in an experimental model of acute ischemia of myocardium demonstrated a relationship between $\mathrm{T}_{\mathrm{E}-\mathrm{Em}}$, deterioration of $\mathrm{LV}$ diastolic function and a subsequent increase of LV filling pressures during acute myocardial ischemia ${ }^{19}$.

Diwan et al. confirmed these results in a clinical study demonstrating a significant inverse correlation of invasively measured LV filling pressures and time interval $\mathrm{T}_{\mathrm{E}-\mathrm{Em}}$ $\left(\right.$ ref. $\left.^{20}\right)$. However, correlation of the time interval $\mathrm{T}_{\mathrm{E}-\mathrm{Em}}$ needs to be considered an academic demonstration of a narrow pathophysiologic connection between ischemia of LV myocardium and an early phase of diastole. A concept of myocardial ischemia inducing a lag of an early annular mitral motion can generally be proposed. Myocardial relaxation in early phase of diastole is energetically very demanding $^{22-24}$. It leads to a diastolic LV dysfunction with increased enddiastolic LV pressures. Correlation of $\mathrm{T}_{\mathrm{E}-\mathrm{Em}}$ to the invasively measured LV filling pressures is however very loose. This is in an agreement with a study published by Guron et al. ${ }^{21}$. Presently there is no study available describing a correlation of $\mathrm{T}_{\mathrm{E}-\mathrm{Em}}$ and an improvement in systolic LV function after revascularization of myocardium. An interpretation of such a relationship is not easy, but in the light of experimental and clinical studies considering hemodynamics and diastolic function of $\mathrm{LV}$, it seams that $\mathrm{T}_{\mathrm{E}-\mathrm{Em}}$ is an indirect echocardiographic marker of hibernating myocardial tissue extent. A viable myocardium is partially able to adaptively react to a chronic coronary hypoperfusion state with inhibition of not only an active contraction of the given hibernating segment but also of an energetically demanding early diastolic relaxation of myocardium in the particular region ${ }^{22-24}$. From this point of view $\mathrm{T}_{\mathrm{E}-\mathrm{Em}}$ is in our study a single echocardiographic parameter predicting revascularization result in terms of reverse LV remodeling onset. It can also be considered an indirect marker of hibernating tissue extent. $\mathrm{T}_{\mathrm{E}-\mathrm{Em}}$ has in prediction of LV reverse remodeling in our study sensitivity $78 \%$ and specificity $78 \%$. However, this parameter has to be validated mainly according to reproducibility, which is relatively favorable in our study $\left(\mathrm{T}_{\mathrm{E}-\mathrm{Em}}\right.$ interobserver and intraobserver coefficient of variation $7 \%$ and $5 \%$ respectively). Another weak point of this parameter is the presence of atrial fibrillation leading to an inhomogeneity of individual heart cycle lengths. This particular problem can be partly solved by measuring $\mathrm{T}_{\mathrm{E}-\mathrm{Em}}$ after a selection of heart cycles with identical R-R interval. A small number of patients with atrial fibrillation in our study do probably not distort results of this parameter measurement.

\section{Limitations}

The mentioned results need to be verified on a larger number of patients in a similarly designed multicentric study, which definitely proves or refutes advisability of measuring some baseline parameters in term of selection of an optimal treatment strategy in a group of patients with ischemic cardiomyopathy and LV systolic dysfunction.

\section{CONCLUSIONS}

After 11 moths from revascularization in patients with a preoperative documentation of viability of myocardium, there is an increase in LVEF, decrease in EDV and ESV. From echocardiographic parameters, $\mathrm{T}_{\mathrm{E}-\mathrm{Em}}$ is potentially usable. This parameter solely predicts reverse LV remodeling after revascularization of myocardium. Values of $\mathrm{T}_{\mathrm{E}-\mathrm{Em}}$ can be an indirect marker of a nonviable hibernating myocardial tissue extent. Fixed perfusion defect extent representing a nonviable myocardial tissue assessed using gated-SPECT of myocardium is an important preoperative predictor of LV function improvement after revascularization. It is necessary to approve results of this study concerning revascularization outcome prediction on a larger amount of patients in a similarly designed multicentric study.

\section{REFERENCES}

1. American Heart Association. Heart and Stroke Statistics: 2004 Update. Dallas, Texas: American Heart Association; 2003. http:// www.americanheart.org

2. Gheorghiade M. Chronic heart failure in the United States: a manifestation of coronary artery disease. Circulation. 1998; 97:282289.

3. Gheorghiade M., Sopko M, De Luca L. et al. Navigating the Crossroads of Coronary Artery Disease and Heart Failure. Circulation. 2006; 114:1202-1213. 
4. Salomon SD, Anavekar N, Skali H et al. Influence of Ejection Fraction on Cardiovascular Outcomes in a Broad Spectrum of Heart Failure Patients. Circulation 2005; 112:3738-3744.

5. Turina J, Turina M, Rothlin $\mathrm{M}$ et al. Improved late survival in patients with chronic aortic regurgitation by earlier operation. Circulation 1984; 70(I):147-52.

6. Gaasch WH, Schick EC. Symptoms and left ventricular size and function in patients with chronic aortic regurgitation. J Am Coll Cardiol 2003; 41:1325-8.

7. Sharir T, Germano G, Kavanagh PB, et al. Incremental prognostic value of post-stress left ventricular ejection fraction and volume by gated myocardial perfusion single photon emission computed tomography. Circulation 1999; 100:1035-1042.

8. Chareonthaitawee P. Revascularization in Severe Left Ventricular Dysfunction: The Role of Viability Testing. JACC, 2005; 46:567574.

9. Bax JJ, van der Wall EE, Harbinson M. Radionuclide techniques for the assessment of myocardial viability and hibernation. Heart 2004; 90(Suppl 5):26-33.

10. Lang RM, Bierig M, Devereux RB et al. Recommendations for Chamber Quantification: A Report from the American Society of Echocardiography's Guidelines and Standards Committee and the Chamber Quantification Writing Group, Developed in Conjunction with the European Association of Echocardiography, a Branch of the European Society of Cardiology. J Am Soc Echocardiogr 2005; 18:1440-1463.

11. Allman KC, Shaw LJ, Hachamovitch et al. Myocardial viability testing and impact of revascularization on prognosis in patients with coronary artery disease and left ventricular systolic dysfunction: a meta-analysis. J Am Coll Cardiol 2002; 39:1151-8.

12. Lombardo A, Loperfido F, Trani C et al. Contractile reserve of dysfunctional myocardium after revascularization: a dobutamine stress echocardiography study. J Am Coll Cardiol 1997; 30:63340.

13. Ragosta M, Beller GA, Watson DD et al. Quantitative planar restredistribution ${ }^{201} \mathrm{Tl}$ imaging in detection of myocardial viability and prediction of improvement in left ventricular function after coronary bypass surgery in patients with severely depressed left ventricular function. Circulation 1993; 87:1630-41.

14. vom Dahl J, Altehoefer C, Sheehan FH et al. Recovery of regional left ventricular dysfunction after coronary revascularization. Impact of myocardial viability assessed by nuclear imaging and vessel patency at follow-up angiography. J Am Coll Cardiol 1996 28:948-58.
15. Miller W, Hodge DO, Tointon SK et al. Relationship of Myocardial Perfusion Imaging Findings to Outcome of Patients With Heart Failure and Suspected Ischemic Heart Disease. Am Heart J, 2004; 147(4):714-720.

16. Hřilund-Carlsen PF, Johansen A, Christensen HF et al. Myocardial perfusion scintigraphy as a gatekeeper for invasive procedures. Potential impact of myocardial perfusion scintigraphy as gatekeeper for invasive examination and treatment in patients with stable angina pectoris: observational study without post-test referral bias. Eur Heart J 2006; 27(1):29-34.

17. Garcia MJ, Rodriguez L, Ares M, Griffin BP, Thomas JD, Klein AL. Differentiation of constrictive pericarditis from restrictive cardiomyopathy: assessment of left ventricular diastolic velocities in longitudinal axis by Doppler tissue imaging. J Am Coll Cardiol 1996; 27:108-114.

18. Hasegawa H, Little W, Ohno M et al.: Diastolic mitral annular velocity during the development of heart failure. J Am Coll Cardiol 2003; 41:1590-1597.

19. Rivas-Gotz C, Khoury DS, Manolios M et al. Time interval between onset of mitral inflow and onset of early diastolic velocity by tissue Doppler: a novel index of left ventricular relaxation: experimental studies and clinical application. J Am Coll Cardiol 2003; 42:14631470.

20. Diwan A, McCulloch M, Lawrie GM et al. Doppler estimation of left ventricular filling pressures in patients with mitral valve disease. Circulation. 2005; 111:3281-3289.

21. Guron CW, Persson A, Wikh R and Caidahl K. Can the left ventricular early diastolic tissue-to-blood time interval be used to identify a normal pulmonary capillary wedge pressure? EUJE, Volume 8 (March 2007), Issue 2:94-101.

22. Bowers KC, Allshire AP, Cobbold PH. Bioluminescent measurement in single cardiomyocytes of sudden cytosolic ATP depletion coincident with rigor. J Mol Cell Cardiol. 1991; 24:213-218.

23. Tian R, Christie ME, Spindler M, et al. Role of MgADP in the development of diastolic dysfunction in the intact beating heart. J Clin Invest. 1997; 99:745-751.

24. Varma N, Morgan JP, Apstein CS. Mechanisms underlying ischemic diastolic dysfunction: relation between rigor, calcium homeostasis, and relaxation rate. Am J Physiol Heart Circ Physiol. 2003; 284:758-771.

25. Gibbons, RJ, Valeti US, Araoz PA, et al. The Quantification of Infarct Size. J Am Coll Cardiol 2004; 44:1533-42.

26. Wu ZW, Tadamura1 E, Yamamuro M et al. Comparison of ContrastEnhanced MRI with 18F-FDG PET/201Tl SPECT in Dysfunctional Myocardium: Relation to Early Functional Outcome After Surgical Revascularization in Chronic Ischemic Heart Disease. J Nucl Med 2007; 48:1096-1103. 